\title{
On filter boundary conditions in topology optimization
}

\author{
Clausen, Anders; Andreassen, Erik
}

Published in:

Structural and Multidisciplinary Optimization

Link to article, DOI:

10.1007/s00158-017-1709-1

Publication date:

2017

Document Version

Peer reviewed version

Link back to DTU Orbit

Citation (APA):

Clausen, A., \& Andreassen, E. (2017). On filter boundary conditions in topology optimization. Structural and Multidisciplinary Optimization, 56, 1147-1155. https://doi.org/10.1007/s00158-017-1709-1

\section{General rights}

Copyright and moral rights for the publications made accessible in the public portal are retained by the authors and/or other copyright owners and it is a condition of accessing publications that users recognise and abide by the legal requirements associated with these rights.

- Users may download and print one copy of any publication from the public portal for the purpose of private study or research.

- You may not further distribute the material or use it for any profit-making activity or commercial gain

- You may freely distribute the URL identifying the publication in the public portal

If you believe that this document breaches copyright please contact us providing details, and we will remove access to the work immediately and investigate your claim. 


\title{
On filter boundary conditions in topology optimization
}

\author{
Anders Clausen · Erik Andreassen
}

Received: date / Accepted: date

\begin{abstract}
Most research papers on topology optimization involve filters for regularization. Typically, boundary effects from the filters are ignored. Despite significant drawbacks the inappropriate homogeneous Neumann boundary conditions are used, probably because they are trivial to implement. In this paper we define three requirements that boundary conditions must fulfill in order to eliminate boundary effects. Previously suggested approaches are briefly reviewed in the light of these requirements. A new approach referred to as the "domain extension approach" is suggested. It effectively eliminates boundary effects and results in well performing designs. The approach is intuitive, simple and easy to implement.
\end{abstract}

Keywords Topology optimization · Filter boundary conditions $\cdot$ Minimum length scale

\section{Introduction}

The goal of topology optimization is to optimally distribute material in a predefined physical domain. Bearing this aim in mind it is remarkable how boundary effects for elasticity problems such as minimum compliance or compliant mechanism design are neglected in the vast majority of research papers on topology optimization. Boundary effects are a consequence of using filters to regularize the design problem. For elasticity problems most publications more or less consciously apply homogeneous Neumann boundary conditions

The authors acknowledge support from the Villum Foundation (the NextTop project) and DTU Mechanical Engineering.

A. Clausen (ac@adimant.com)·E. Andreassen

Department of Mechanical Engineering, Solid Mechanics,

Technical University of Denmark, Nils Koppels Alle, B. 404,

DK-2800 Lyngby, Denmark
(BCs) at all domain boundaries. At a symmetry condition this choice of BCs is mathematically and physically correct. However, for other cases the reason for using these BCs seems to be that they are trivial to implement. They come automatically if no modifications to filter operators are performed, and they give visually "nice" results for standard benchmark problems.

As an example, consider the MBB beam benchmark problem (Fig. 19) optimized with the robust approach (Wang et al, 2011) using homogeneous Neumann BCs on the filter. The robust approach is briefly described in Section 2 It should be stressed, however, that the BC issues do not only occur for the robust formulation but are of general concern. The optimized design suffers from three issues, indicated by numbered arrows in the figure: (1) The minimum feature size is not satisfied along domain boundaries; (2) structural edges are forced to be perpendicular to domain boundaries; and (3) the structure appears to "stick" to the domain boundaries. In the von Mises stress plot at right, the lowly stressed regions at sharp corners reveal excess material. Note that similar features are also seen for other filtering strategies.

Issues 1 and 2 are a consequence of the homogeneous Neumann BCs effectively acting as a symmetry condition. This appears clearly if locally mirroring the optimized design (Fig. 1 b). Issue 3 is driven by two circumstances: first, since structural edges at an external boundary are not filtered with void (Fig. 1, ), the expensive, penalized intermediate densities are avoided at early design iterations when the projection is less sharp; second, since structural edges are forced to be perpendicular to the boundary (issue 2), structural members cannot curve smoothly away from the boundary.

Based on these observations it is clear that homogeneous Neumann BCs are a suboptimal choice for the robust optimization approach and more broadly for elasticity based topology optimization. Some flaws have already been pointed 


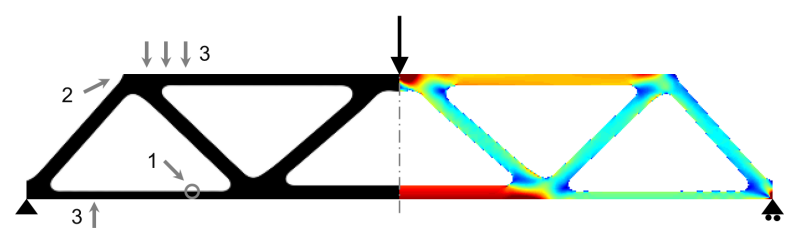

(a)
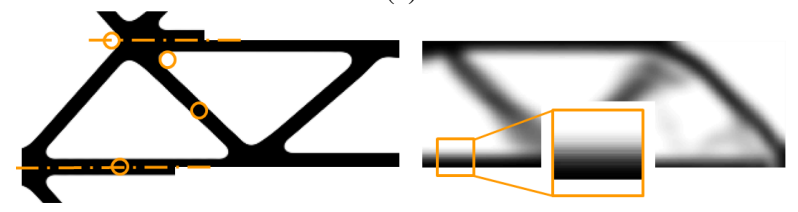

(b)

(c)

Fig. 1: Example MBB benchmark problem optimized with homogeneous Neumann BCs on the filter. (a) Optimized structure with von Mises stresses on mirrored part. Issues indicated by numbered arrows. (b) Locally mirrored structure indicating consequences of the symmetry condition on the filter. (c) External structural edge is not filtered with void.

out in earlier works by Lazarov et al (2011, 2016), and several alternatives have been suggested to remedy the issues. However, no convincing systematic approach to eliminate boundary effects has yet been suggested. The authors wish to underline that this paper addresses an issue with general relevance for topology optimization problems. The issue is not limited to density-based approaches.

The novelty of this work is threefold: (1) Give a clear and precise definition of the (non-physical) domain boundary effects caused by filtering schemes; (2) present a rigorous method which solves the issue of (non-physical) boundary effects; and (3) as a side effect of solving this issue, present a robust design method which assures the same minimum length scale at the boundary as in the interior of the design domain.

The remainder of the paper is structured as follows: First a brief definition of the problem setting is presented. Then three requirements are suggested that the ideal BC approach should fulfill, and previously suggested BCs are discussed in the light of these requirements. After this, the approach suggested by the present work, the domain extension approach, is presented. The suggested approach is supported by both qualitative and quantitative comparisons with the homogeneous Neumann conditions for the MBB beam and the force inverter benchmark problems.

For simplicity only the $2 \mathrm{D}$ problem is considered, but the methodology is fully applicable in $3 \mathrm{D}$.

\section{Problem setting}

The vast majority of topology optimization approaches make use of some sort of filters for regularization. This is true not only for density-based methods, but also in phase field and level-set approaches. For a general review of the different topology optimization approaches see e.g. (Sigmund and Maute, 2013). The most simple approaches only involve a single, basic filter (Sigmund, 2001; Andreassen et al, 2011), such as the sensitivity filter (Sigmund, 1994, 1997) or the density filter (Bruns and Tortorelli, 2001; Bourdin, 2001), potentially in the form of a PDE-based filter (Lazarov and Sigmund, 2011). A density-based filter may be followed by a projection step (Guest et al, 2004, Xu et al, 2010) to force the design towards a $0 / 1$ solution. The considerations presented in this paper are relevant for all of these filters.

The most important flaw of the homogeneous Neumann $\mathrm{BCs}$, however, is the violation of the minimum feature size at the domain boundary. A detailed review of feature size control and manufacturability is presented by Lazarov et al (2016). In the present paper, the main focus will be on the robust formulation (Sigmund, 2009, Wang et al, 2011). The robust method works by considering not only the optimized "blueprint" design, but also an eroded and a dilated version. For a design problem governed by linear elasticity, the method may be formally defined in the following way:

$$
\min _{\boldsymbol{\rho}} \max \left(c_{\mathrm{b}}, c_{\mathrm{e}}, c_{\mathrm{d}}\right)
$$

subject to: $\quad \mathbf{K}\left(\overline{\tilde{\boldsymbol{\rho}}}_{b}\right) \mathbf{U}_{b}=\mathbf{F}$,

$$
\mathbf{K}\left(\overline{\tilde{\boldsymbol{\rho}}}_{e}\right) \mathbf{U}_{e}=\mathbf{F} \text {, }
$$$$
\mathbf{K}\left(\overline{\tilde{\boldsymbol{\rho}}}_{d}\right) \mathbf{U}_{d}=\mathbf{F},
$$$$
g\left(\overline{\tilde{\boldsymbol{\rho}}}_{d}\right)=V\left(\overline{\tilde{\boldsymbol{\rho}}}_{d}\right) / V^{*}-1 \leq 0,
$$

$$
0 \leq \rho_{i} \leq 1, \quad \forall i
$$

where $\boldsymbol{\rho}$ is the vector of design variables, $c$ is the objective, subscripts $b, e$ and $d$ indicate the blueprint, eroded and dilated designs, respectively, $\mathbf{K}$ is the global stiffness matrix, $\overline{\tilde{\boldsymbol{\rho}}}$ is the vector of filtered and projected densities, $\mathbf{U}$ and $\mathbf{F}$ are the global displacement and force vectors, respectively, $g$ is the volume constraint, $V$ is the material volume, $V^{*}$ is the maximum allowed volume, and $i$ is the element index. The volume constraint is applied on the dilated field, but is adjusted such that the final volume on the blueprint design corresponds to the design specification. For the standard minimum compliance problem, $c$ indicates the compliance, and the eroded design will always have the highest objective value. For a mechanism design problem, the objective function to optimize is an output displacement, $u_{\text {out }}$. The blueprint, eroded, and dilated structures are modeled through a projection step with varying threshold, typically $\eta=0.5$ for the blueprint design and $\eta_{d}<\eta<\eta_{e}$ for the dilated and eroded designs, respectively. Under the assumption that the three designs have identical topology, the robust approach ensures a minimum length scale. With homogeneous Neumann BCs, however, the feature size is not ensured at the boundary as described in Section 1 . 
Since homogeneous Neumann conditions eliminate spatial gradients at the domain boundary, also the approach of imposing a minimum feature size using explicit constraints based on gradients (Zhou et al, 2015) will need modifications at the boundary.

Local length scale control has also recently been suggested for the level-set approach (Allaire et al, 2016, Wang et al, 2016). Also for this framework, filter/regularization BCs influence the design, both with and without thickness constraints. How striking the BC issues appear depends on whether the minimum length scale is also imposed on the void region. However, it will always be noticeable in the objective function-if the choice of filtering BCs tricks or forces the optimization to put material in the wrong place, the optimized part will have a decreased performance. This point is particularly relevant in material restricted problems.

In the following the term "design domain" refers to the domain within which the designer is allowed to place the final, optimized structure. For a standard filtering-projection scheme or the robust formulation, the domain of mathematical design variables is coincident with the design domain if the projection threshold is chosen as $\eta=0.5$ for the blueprint design. For other choices of filtering schemes such as a density or sensitivity filter alone, the final design will contain gray elements at the interface between solid and void. Before manufacturing, an interpretation to a fully solid/void design must be performed. This will typically be done by means of some contour extraction method based on the 0.5 level-set curve. Also in this case the domain of mathematical design variables is coincident with the design domain. For more complex filtering schemes, e.g. (Clausen et al, 2015), the two domains may not be coincident.

The terms "Dirichlet" and "Neumann" BCs will throughout this paper refer to the filter BCs only. BCs for the finite element (FE) problem will not be described using these terms in order to avoid confusion.

\section{Approach}

\subsection{Requirements to the ideal approach}

The issues observed when using homogeneous Neumann BCs lead us to define three requirements which the ideal approach should fulfill in order to eliminate boundary effects:

1. External vs. internal edges: External structural edges should be treated exactly like internal ones.

2. Length scale control: The minimum allowed feature size should not be affected by boundary effects.

3. Restriction in space: The optimized structure cannot exceed the predefined physical design domain. On the other hand it should be possible to place features of any allowed size all the way to the domain boundary.

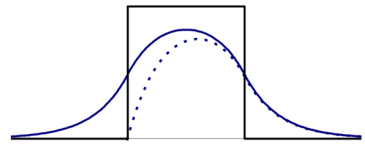

(a)

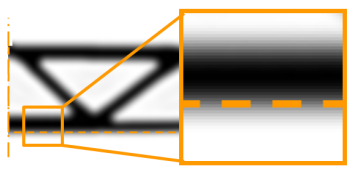

(b)
Fig. 2: Filtering with other BCs than homogeneous Neumann. (a) Filtering a sharp-edged feature using a void padding (solid blue) or a homogeneous Dirichlet BC (dotted). (b) Gray elements outside the design domain as a result of using a void padding. Compare to Figure 1 .

The term "external structural edges" here refers to edges sufficiently close to the domain boundary to be affected by potential boundary effects.

\subsection{Previous alternatives}

Apart from the homogeneous Neumann BCs discussed in the introduction, two other types of filter BCs have been suggested in the literature: using Dirichlet conditions; or using a boundary padding on the filter domain. These two alternative approaches are discussed below.

\subsubsection{Dirichlet conditions}

Lazarov et al (2011) pointed out the issue that the minimum feature size is only half the width at the domain boundary when using homogeneous Neumann BCs. The paper suggested using a PDE-filter (Lazarov and Sigmund, 2011) with Dirichlet BCs as an alternative to the homogeneous Neumann BCs. The Dirichlet BCs were chosen as nonhomogeneous at loads/supports and homogeneous elsewhere (except at symmetry conditions). Clausen et al (2015) employed the PDE-filter with a similar choice of BCs, and Lazarov et al (2016) pointed out Dirichlet BCs as an option. All three papers suggest using a filter support domain which is coincident with the design domain.

The choice of Dirichlet BCs is physically meaningful and removes the symmetry condition imposed by homogeneous Neumann BCs. However, the approach violates Requirement 1 as stated above, in that external structural edges are treated differently than internal edges: A symmetric feature in the design field located sufficiently close to the domain boundary becomes non-symmetric after filtering (Figure 2). Two concrete consequences is that a bar at the boundary will not have coincident center lines for the dilated, eroded and blueprint structures, and that the spacial gradient field will behave differently, which is a problem in approaches using this field, e.g. (Clausen et al, 2015).

Dirichlet BCs furthermore imply a violation of the subpoint in Requirement 3: If the projection threshold is $\eta=0.5$ 
and a homogeneous Dirichlet condition is applied, the projected structural edge cannot be placed exactly at the domain boundary (again, see Fig. 2). Simply enforcing the Dirichlet condition at some non-zero distance outside the domain boundary will not solve this issue, since the distance between the enforced Dirichlet condition and the edge of a projected feature will vary with the feature size. Such a remedy would also spoil the simplicity and physical reasoning, and may pose problems at interface points between homogeneous and nonhomogeneous BCs. Furthermore, it is unclear to the authors if the minimum feature size is guaranteed close to the boundary.

\subsubsection{Filter boundary padding}

An alternative to Neumann or Dirichlet BCs, suggested by e.g. Zhou et al (2014) and Lazarov et al (2016), is to use a boundary padding on the filter, i.e. extend the filter domain beyond the design domain by a distance equal to the filter radius. The field value in the padding before performing the filtering would typically be set in a way similar to the Dirichlet conditions, i.e. solid at loads/supports and void elsewhere. At a symmetry condition homogeneous Neumann $\mathrm{BCs}$ are applied without a padding.

While using a padding alleviates the issues directly related to the symmetry condition of homogeneous Neumann $\mathrm{BCs}$ it still violates Requirement 1, though not as severely as the Dirichlet BCs. This may be seen from Figure $2 \mathrm{~b}$. The filtered field may contain regions of non-zero density $(\tilde{\rho} \in[0,0.5[)$ outside the design domain. If these regions are ignored and not included in the FE evaluation and the volume constraint, gray elements are excluded, and external edges will be favored compared to internal ones.

When using the robust optimization approach it is a particularly rough assumption to ignore gray elements in the filters' boundary padding, as the dilation operation projects low density elements to fully solid density. Consider for instance a feature of width $R$ at the boundary in the design field which is filtered with a linear density filter of radius $R$ and projected with infinite sharpness $(\beta \rightarrow \infty)$ at a threshold of $\eta_{d}=0.25$. For the resulting physical, solid feature, $18 \%$ of the width and thereby axial stiffness exceeds the design domain.

More generally speaking, if a boundary padding is used for the filtering step, but the FE domain is not extended to include the resulting gray elements, boundary effects are introduced. Depending on the problem and approach, this approach may ignore significant stiffness and volume contributions from outside the physical design domain.

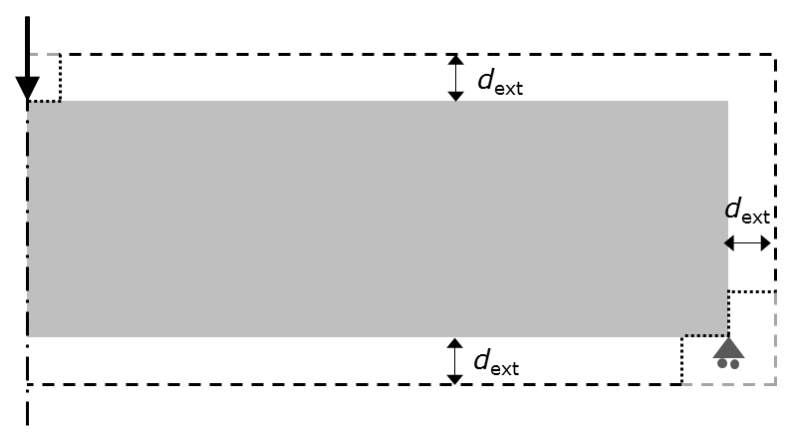

Fig. 3: The domain extension approach. The gray area shows the extent of the design variable field. All other domains are extended to the dashed line. Dotted lines indicate local modifications of the domain extension at loads and supports. All external boundaries have homogeneous Neumann BCs.

\subsection{The domain extension approach}

The domain extension approach builds on the same idea as using a boundary padding for the filter. However, due to the issues described in the preceding section, it is critical to extend not only the filter domain, but also the FE domain. This leads to the definition of the approach suggested in this work, which we refer to as the "domain extension approach". If we initially ignore the need for local modifications at loads and supports, the idea of the approach may be stated as follows: The spatial extent of the blueprint design should be limited by the extent of the design variable field; all other domains used for filters, FEA, gradient field computation etc. should be extended sufficiently beyond the boundaries of the design domain to avoid boundary effects. The approach is illustrated in Figure 3, where the gray area corresponds to the design variable field and the dashed line indicates the boundary of the extended domains. The distance $d_{\text {ext }}$ should typically be at least one filter radius. The extension is performed by adding a padding of void elements to the design domain before the first filter operation. At symmetry conditions homogeneous Neumann BCs are applied without a padding. The final blueprint design is defined by the physical densities inside the design domain.

For a linear filter or other filters with finite support it makes no difference which BCs are applied at the external boundary of the padding if the padding width, $d_{e x t}$, is at least the filter radius. For the PDE-filter, which in theory has infinite support, the choice does influence the filtered field, however, numerical experiments have shown that for all practical purposes it is sufficient to use a padding width equal to the filter radius, and that it makes no difference whether Dirichlet or homogeneous Neumann conditions are used. We therefore suggest using the simple homogeneous Neumann BCs at the extended boundary. 


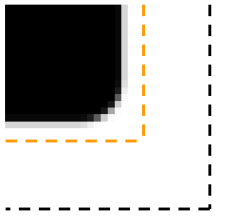

(a)

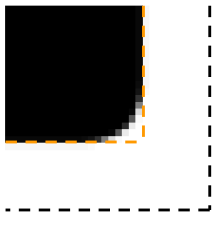

(b)

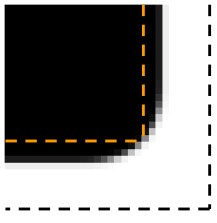

(c)
Fig. 4: Illustration of the need for local modifications of the domain extension approach, if loads and supports are placed close to the domain boundary. The boundary is indicated by the dashed, orange line, and the extension by the dashed, black line. When filtering a fully solid design domain with a void padding, the edges of the (a) eroded, (b) blueprint, and (c) dilated structures are not aligned. Without local modifications, the FE problem may become singular.

Loads or supports defined at or close to the design domain boundary require minor, local modifications to the domain extension. Otherwise, the method will fail since the eroded field defined by a threshold $\eta_{e}>0.5$ will never reach the boundary (Figure 4). As a result, the FE problem may become singular. Also for problems which do not employ the robust approach, local modifications may be needed: Since the design domain is filtered with a void padding, boundary elements will have intermediate density at early design iterations where the projection sharpness is low. This implies high local displacements at loaded or supported points. If the support or load is placed in the corner, the modification is mandatory (see Figure $4 \mathrm{~b}$ ). We therefore suggest that the domain extension is omitted at loaded or supported points closer to the domain boundary than one filter radius. This modification is indicated by dotted lines in Figure 3. Instead, homogeneous Neumann BCs are applied directly at the non-extended boundary. Thereby the described issues are removed. The region of the domain boundary which is not extended should be consistent with the minimum feature size and not smaller than one filter radius in any direction along the boundary. At the interface between the nonextended and extended parts of the domain, the extension should be performed perpendicularly on the boundary. Note that this modification implies that the minimum feature size is not guaranteed at loads and supports, however, if any issues appear they may easily be alleviated by adding a block of solid, passive elements.

As follows from the above justifications, the domain extension approach allows the blueprint design to exceed the design domain with gray densities for a finite projection sharpness. This is, however, no problem as long as the final design is a black and white interpretation of the grayscale design. The dilated design interpreted as a black and white structure will obviously still exceed design domain. Again this is no problem. If the designer wants a margin for manu- facturing uncertainty this margin would in any case require an additional restriction of the design domain.

For some problem formulations, the simplified technique of using a void padding on the filter and ignoring gray elements in the padding for the FE problem, as done by Zhou et al (2014) and Lazarov et al (2016), may lead to similar results. An example is compliance problems optimized with the robust approach, where it is sufficient to optimize the eroded design. However, the choice of ignoring stiffness and volume contributions in the boundary padding should always be stated explicitly and justified.

\section{Validation}

\subsection{Basis for comparison}

As described in Sections 1 and 3.2 the three previously employed choices of BCs, i.e. the standard homogeneous Neumann approach, the Dirichlet approach and the boundary padding approach, all violate the requirements put forth in Section 3.1 and introduce boundary effects. However, for illustration purposes the domain extension approach is here compared with the corresponding structures obtained with homogeneous Neumann BCs.

The minimum feature size requirement is a heavy performance limitation, and since the standard homogeneous Neumann conditions permit violating the minimum feature size, it does not make sense to quantitatively compare the two approaches directly. However, with a minor modification to the homogeneous Neumann BCs the minimum feature size can be assured to be satisfied and the methods can be more directly compared. This modification is described in the following.

Consider a density filter with a constant radius $R$ in the interior of the filter domain. Recall that the homogeneous Neumann BCs effectively act as a symmetry condition at the boundary. Therefore, by locally modifying the filter radius at the boundary from $R$ to $2 R$, an effective minimum feature size is obtained for the solid phase, corresponding to using a radius of $R$ in the interior. By letting the local radius decay linearly from $2 R$ to $R$ at a distance of $R$ from the boundary a smooth transition is obtained. The modification of the local radius is only performed in the direction normal to the domain boundary and thus results in a locally anisotropic filter.

With this modification, Requirement 2 is fulfilled for the solid phase. However, Requirement 1 is not satisfied with this approach since it still forces structural edges to be perpendicular to domain boundaries, now amplified to a local radius in the void domain of $2 R$.

This minor modification will be used in the performance comparison and labeled "homogeneous Neumann BCs with anisotropic filter". 


\subsection{Benchmark problems}

Two benchmark problems are considered to validate the domain extension approach: The MBB beam and the force inverter. For the MBB beam the design domain dimensions are $300 \times 100$ and the filter radius is $R=10$. The domain is discretized with square unit length bi-linear elements. The projection thresholds are $\eta=0.5, \eta_{d}=0.25$ and $\eta_{e}=0.75$ for the blueprint, dilated and eroded designs, respectively.

The penalization is omitted, i.e. $p=1$. Instead black and white designs are ensured by using a continuation approach on the projection, starting at $\beta=1$, gradually increasing by a factor of $\sqrt{2}$ at every 50 iterations up to $\beta_{\max }=32$. The maximum allowed number of iterations is 1000 . All compliance values refer to thresholded designs, with the final threshold value chosen individually for each optimized structure such that the volume constraint is exactly satisfied. The figures show the non-thresholded designs, illustrating how intermediate densities are practically absent.

Figure $5 \mathrm{a}$ shows the MBB beam optimized with a volume constraint of $40 \%$ for three different filter approaches: the standard homogeneous Neumann approach, the homogeneous Neumann approach with anisotropic filter, and the domain extension approach. As opposed to the standard Neumann approach, the other two approaches satisfy the minimum feature size. The compliance values are 233.4, 236.7 and 233.8, respectively. That is, the domain extension approach performs $1.2 \%$ better than the homogeneous Neumann approach with anisotropic filter, and almost exactly like the standard homogenous Neumann approach in spite of the latter not satisfying the minimum length scale. Stress concentrations at loads and supports are in the plot shown with a maximum value of 0.35 . A zoom at the boundary for the MBB beam optimized using the domain extension approach (Figure 5b) confirms that the structure does not exceed the physical design domain.

Figure 6 compares MBB beam results obtained at varying volume constraint value using homogeneous Neumann BCs with anisotropic filter and the domain extension approach (the two only approaches from Figure 5 which satisfy the minimum feature size). The domain extension approach performs consistently slightly better in terms of compliance $(0.8-1.2 \%)$ and eliminates the peculiarities at the domain boundary.

The structures obtained with the domain extension approach in Figure 6 no longer have a straight connection between the two supports. Instead of being parallel to the lower domain boundary, the lower bar closest to the support is sloped slightly from the horizontal direction and joins the other bar above the supported point. Rather than an undesirable boundary effect, the authors believe that this difference compared to the structures obtained with the homogeneous Neumann BC structures is a result of a trade-off between sat-

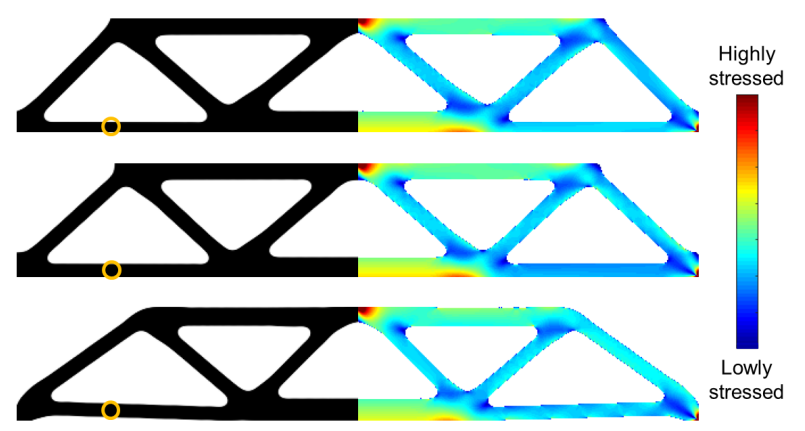

(a)

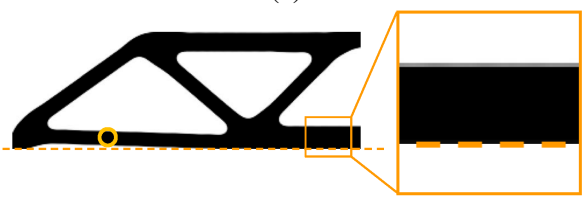

(b)

Fig. 5: (a) MBB beam optimized with the standard homogeneous Neumann BCs (top), homogeneous Neumann BCs with anisotropic filter (middle) and domain extension approach (bottom). Mirrored part illustrates the von Mises stresses (colorbar: $[0,0.35]$ ). (b) Zoom at the boundary of the structure optimized using the domain extension approach.

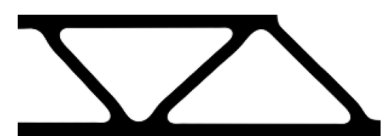

$c=330.4$

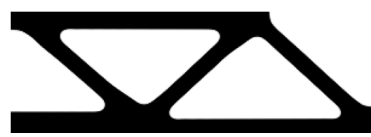

$c=236.7$

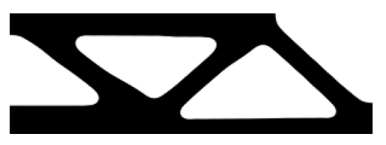

$c=196.2$

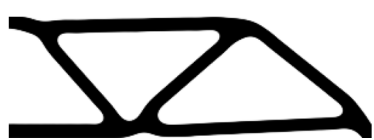

$c=326.4$

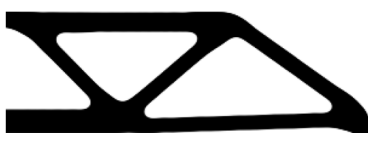

$c=233.8$

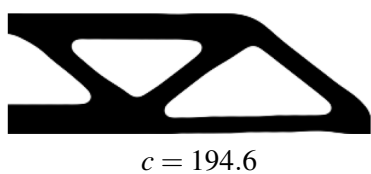

Fig. 6: MBB beam optimized with homogeneous Neumann BCs with anisotropic filter (left) and domain extension approach (right). Volume fractions (from the top): $30 \%, 40 \%$ and $50 \%$.

isfying the minimum feature size and the volume constraint. This also explains why the effect becomes increasingly pronounced for decreasing volume fraction-for the $30 \%$ volume design, all features have nearly equal thickness meaning that the design freedom is quite restricted. Note also, that had it been an undesirable boundary effect, one would expect the $30 \%$ volume design, for which the effect is most pronounced, to perform relatively worse compared to the homogeneous Neumann approach than the designs obtained at a higher volume fraction where the effect is less apparent. 
However, this is not the case. Specifically, the non-straight lower bar is not a result of the omitted domain extension at the support, since it also appeared for test examples where the domain extension was instead modified at the support by using passive solid blocks (not included in the article).

Compliance values do not vary much between the two presented approaches. However, it seems that the classic MBB beam benchmark case, with the supported node defined in the corner of the domain, to some extent conceals the flaws of the homogeneous Neumann BCs. For this test case, the tendency of placing material along the bottom edge of the domain follows the straight line between the two supports at the bottom of the domain. Intuition and a number of numerical tests performed with the domain extension approach indicate that this line is the optimal load path in sustaining the horizontal force at the simply supported node.

Slight modifications to the problem reveal the shortcomings of the homogeneous Neumann BCs-Figure 7 shows an example where the issues are clearly exposed. In this problem the support is translated 1/4 of the domain height upwards. Due to the requirement that edges must be perpendicular to the domain boundary, the structure ends up with a highly sub-optimal sharp bend in the middle of a beam, both when using the standard homogeneous Neumann BCs and homogeneous Neumann BCs with anisotropic filter. This artifact is avoided with the extended domain approach. The compliance values are 232.6, 236.4 and 228.0, respectively, i.e. the domain extension approach outperforms the two versions of homogeneous Neumann BCs by $2 \%$ and $4 \%$, respectively.

More generally, it seems that homogeneous Neumann BCs produce dissatisfactory results when the optimal load path goes close to the domain boundary, but is not aligned with it. A related issue to that of Figure 7 is expected to appear if the design domain boundary curves outwards between two points that should optimally be connected by a straight bar, the homogeneous Neumann conditions would either have to place material all along the curved boundary, or at some point make a sharp corner at the middle of a beam, as observed in Figure 7. Neither result would be optimal.

The force inverter problem is solved using the design domain from (Wang et al, 2011) with the parameters from Figure $8(\mathrm{j}-1)$ in the same paper: Domain dimensions are $200 \times$ $100, R=8.4, \eta_{d}=0.2$ and $\eta_{e}=0.8$. The penalization and continuation scheme follow those described above for the MBB problem. The maximum allowed number of iterations is 1500 .

Figure 8 compares the blueprint structures for (a) standard homogeneous Neumann BCs like in the original paper, (b) homogeneous Neumann BCs with anisotropic filter, and (c) the domain extension approach. The horizontal output displacements are $-1.96,-1.71$ and -1.81 , respectively.

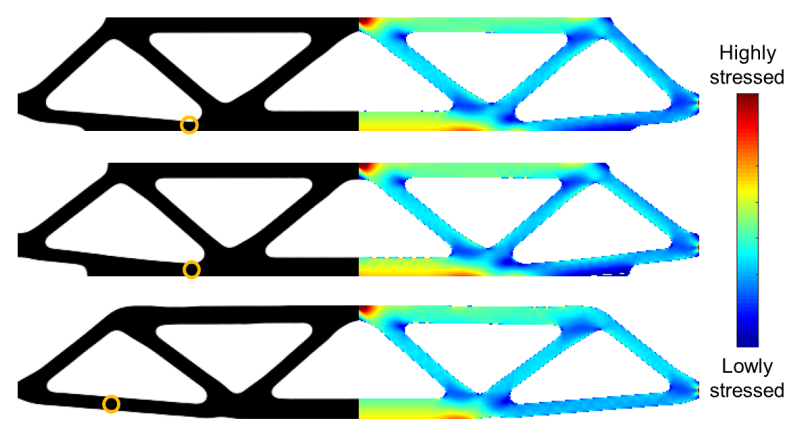

Fig. 7: MBB beam where the support is translated 1/4 of the domain height upwards, using the standard homogeneous Neumann BCs (top), homogeneous Neumann BCs with anisotropic filter (middle), and domain extension approach (bottom). Volume fraction: 40\%. Mirrored part illustrates the von Mises stresses (colorbar: [0, 0.35]).

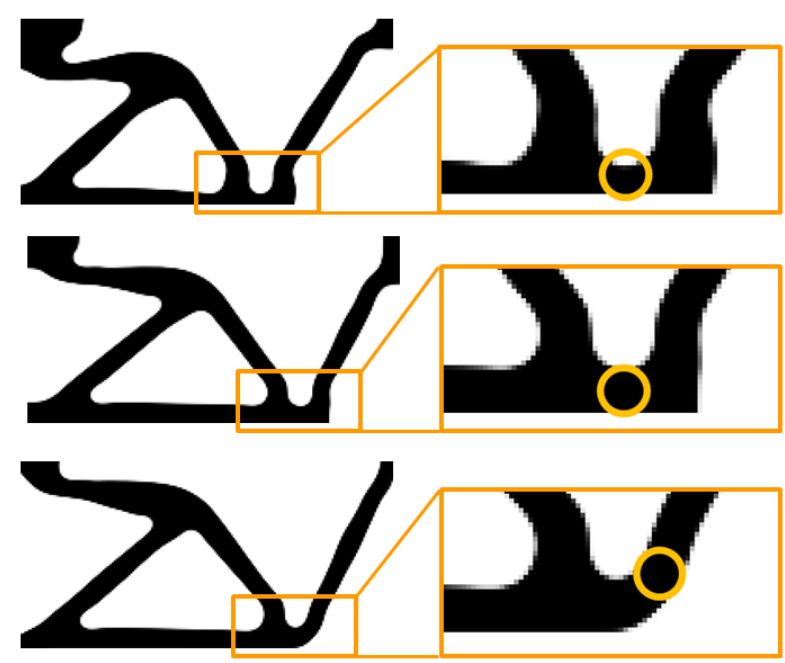

Fig. 8: Optimized blueprint designs for force inverter problem. (a) Homogeneous Neumann BCs. (b) Homogeneous Neumann BCs with anisotropic filter. (c) Domain extension approach. The reason that the structure in (a) differs slightly from the corresponding structure in (Wang et al, 2011) and has a slightly better performance is the modified penalization and continuation scheme.

When inspecting the plots of the optimized structures it is clear that the superior performance obtained with standard homogeneous Neumann BCs is due to a violation of the minimum feature size at the hinge, which is placed at the domain boundary. In similarity with the initial observations for the MBB problem, the algorithm exploits flaws in the choice of BCs. The two latter designs satisfy the minimum feature size. Here the design obtained with the domain extension approach is superior by $6 \%$. 


\section{Conclusions}

This paper presents the domain extension approach to filter boundary conditions for topology optimization problems. Three requirements which filter BCs should comply with in order to eliminate boundary effects are defined. The domain extension approach satisfies all three of them.

The paper furthermore introduces a simple modification to the standard homogeneous Neumann BCs ensuring that the minimum feature size is satisfied everywhere: A locally varying anisotropic filter. This latter method, however, still suffers from the other issues observed with standard homogeneous Neumann conditions.

For the standard MBB beam benchmark case the compliance values of the domain extension approach are only marginally better. For more general minimum compliance design problems, where the domain boundary is not necessarily aligned with the optimal load path, the domain extension approach performs significantly better than the homogeneous Neumann approach. For the force inverter problem, the domain extension approach removes boundary effects from the design of the hinge.

The main drawback of the domain extension approach is that features smaller than the filter radius cannot be placed exactly at the domain boundary (only relevant for values of $\eta_{e}<0.75$ and $\left.\eta_{d}=1-\eta_{e}>0.25\right)$. In this case the sub-point in Requirement 3 is thus slightly violated. However, all other advantages are kept, independently of the value of $\eta_{e}$. This drawback is considered of minor importance compared to the advantages of the approach.

In the presence of small internal holes or narrow voids in the physical design domain, the padded areas from opposing sides of the void might overlap. As long as the minimum feature size is also enforced in the void region, and the minimum feature size is larger than or equal to the filter radius, no parts of the padding will overlap the design space. Since the entire padding has zero density the overlap poses no problems for the filter. For the practical domain definition, the user can simply offset the entire boundary and define the extended domain as the boolean union of the original domain and the offset volume.

The discussion in this work only covers elasticity-based problems, however, all elements of the approach can be transfered to a broader range of problems.

Acknowledgements The authors wish to thank the TopOpt research group at the Technical University of Denmark, particularly Professor Ole Sigmund, for valuable discussions on the topic of this paper.

\section{References}

Allaire G, Jouve F, Michailidis G (2016) Thickness control in structural optimization via a level set method. Struc- tural and Multidisciplinary Optimization 53(6):13491382

Andreassen E, Clausen A, Schevenels M, Lazarov BS, Sigmund O (2011) Efficient topology optimization in matlab using 88 lines of code. Structural and Multidisciplinary Optimization 43(1):1-16

Bourdin B (2001) Filters in topology optimization. International Journal for Numerical Methods in Engineering 50(9):2143-2158

Bruns TE, Tortorelli DA (2001) Topology optimization of non-linear elastic structures and compliant mechanisms. Computer Methods in Applied Mechanics and Engineering 190(26-27):3443-3459

Clausen A, Aage N, Sigmund O (2015) Topology optimization of coated structures and material interface problems. Computer Methods in Applied Mechanics and Engineering 290:524-541

Guest J, Prevost J, Belytschko T (2004) Achieving minimum length scale in topology optimization using nodal design variables and projection functions. International Journal for Numerical Methods in Engineering 61(2):238-254

Lazarov BS, Sigmund O (2011) Filters in topology optimization based on helmholtz-type differential equations. International Journal for Numerical Methods in Engineering 86(6):765-781

Lazarov BS, Schevenels M, Sigmund O (2011) Robust design of large-displacement compliant mechanisms. Mechanical sciences 2(2):175-182

Lazarov BS, Wang F, Sigmund O (2016) Length scale and manufacturability in density-based topology optimization. Archive of Applied Mechanics pp 1-30

Sigmund O (1994) Design of material structures using topology optimization. PhD thesis, Department of Solid Mechanics, Technical University of Denmark

Sigmund O (1997) On the design of compliant mechanisms using topology optimization. Journal of Structural Mechanics 25(4):493-524

Sigmund O (2001) A 99 line topology optimization code written in Matlab. Structural and Multidisciplinary Optimization 21(2):120-127

Sigmund O (2009) Manufacturing tolerant topology optimization. Acta Mechanica Sinica 25(2):227-239

Sigmund O, Maute K (2013) Topology optimization approaches. Structural and Multidisciplinary Optimization 48(6):1031-1055

Wang F, Lazarov BS, Sigmund O (2011) On projection methods, convergence and robust formulations in topology optimization. Structural and Multidisciplinary Optimization 43(6):767-784

Wang Y, Zhang L, Wang MY (2016) Length scale control for structural optimization by level sets. Computer Methods in Applied Mechanics and Engineering 305:891-909 
Xu S, Cai Y, Cheng G (2010) Volume preserving nonlinear density filter based on heaviside functions. Structural and Multidisciplinary Optimization 41(4):495-505

Zhou M, Lazarov BS, Sigmund O (2014) Topology optimization for optical projection lithography with manufacturing uncertainties. Applied optics 53(12):2720-2729

Zhou M, Lazarov BS, Wang F, Sigmund O (2015) Minimum length scale in topology optimization by geometric constraints. Computer Methods in Applied Mechanics and Engineering 293:266-282 